\title{
Pen needle pointers
}

\section{Editorial}

Injections are used by many patients with diabetes in the United States. There are currently different classes of injectable medications. The list includes, insulin, Glucagon like Peptide-1 (GLP-1) analogues and amylin analogue. One cause of increased glycemic excursions appears to be poor injection practices. Healthcare professionals play an important role in the optimal use of these medications. Proper injection techniques by patients are essential to achieve good glycemic control, reduce patient discomfort, and improve consistency in medication delivery. Proper injection techniques are rarely taught because of time constraints and possible lack of knowledge. During patient education, healthcare providers and educators concentrate on proper dosing, mixing different insulin's, and the patient's ability to read the pen or the marks on the syringe. Although at some patient education session's injection site rotation is mentioned, there is no mention of types of syringes and needles to choose from. Many educators and providers remain skeptical that shorter needles may benefit their patients, particularly obese patients. Patients receive injection supplies from different vendors and unless providers specify a needle size or syringe gauge, patients receive the cheapest choice of syringe or needle.

To help patients improve compliance with medication regimen, healthcare professionals should spend time with each patient and explore the patient's anxieties about injecting a medication. In addition, the choices and management of devices used has to be discussed with the patient. Current injection practices should be observed including site rotation, injection angle, the possible use of skin folds. In addition, injection site should be examined periodically for signs of lipohypertrophy, inflammation, edema or infection. Decisions regarding optimal needle length, prevention of injection complications, and safe disposal of used sharps should be made in a discussion between the healthcare professional and the patient.

To overcome injection related anxiety, the patient can be consulted that pain from an injection may be reduced if the injected product is kept at room temperature. Factors affecting the patient's perception of pain include needle diameter and length. Using a new needle for each injection, specifically a needle that is shorter with smaller needle diameter, is reported to produce less pain and is more comfortable to use.

Shorter needles, 4-6mm pen needles, can be used by any adult including obese patients without having negative effects on Hemoglobin A1C (HbA1C) levels. In addition, 4-5mm pen needles enter the subcutaneous tissue with minimal risk of intramuscular (IM) injection in virtually all adults. IM injections, which are more common
Volume 3 Issue I - 2016

\section{Samuel Grossman}

Department of Veterans Affairs, American Diabetes Association, USA

Correspondence: Samuel Grossman, Department of Veterans Affairs, American Diabetes Association, USA, Email Samuel.Grossman@va.gov

Received: December 30, 2015 | Published: January 04, 2016

with larger needles, make absorption unpredictable and can increase the risk of hypoglycemia. Injections with shorter needles should be given at a 90 degree angle to the skin surface without a raised skin fold. A raised skin fold should be used in patients that inject into a slim stomach, limbs, or pregnant patients.

Short needle such as the $4 \mathrm{~mm} \times 32 \mathrm{G}$ pen needle known as the Becton Dickinson (BD) Nano shows further benefits compared with longer pen needles and is compatible with all pens sold in the U.S. Patients using shorter needles have less complaints of pain on injection compared with the $31 \mathrm{G} 5 \mathrm{~mm}$ and $8 \mathrm{~mm}$ pen needles. In addition, the $4 \mathrm{~mm} \times 32 \mathrm{G}$ pen needle reduces the risk of IM injection compared with the longer needles over $5 \mathrm{~mm}$. The Nano, for example, allows for a single-handed injection. A straight in injection using a no-pinch technique is more convenient for patients with the added flexibility to inject in the upper arm or the buttocks. The increase in site flexibility provided by the $4 \mathrm{~mm} \times 32 \mathrm{G}$ pen needle facilitates confidence in patients to be more willing to use injectable medication products.

When educating patients, healthcare professionals must emphasize the importance of compliance with medication regimen, correct injection process and proper needle size. Less pain with equivalent glycemic control compared with longer pen needles, ease of use, and compatibility with all pens sold in the U.S makes the Nano an attractive option to reduce anxiety associated with injectable medications. Improved confidence with injections is sure to improve compliance with medication regimen and lead to an improvement in glycemic control.

\section{Acknowledgements}

None.

\section{Conflict of interest}

Author declares that there is no conflict of interest. 